\title{
Sarcoidose na Infância com Manifestações Raras: Vasculite, Acometimento do Sistema Nervoso Central, Ósseo e Genital( ${ }^{*}$
}

\section{Sarcoidosis in Childhood with Rare Manifestations: Vasculitis, Central Nervous System, Bone and Genital Involvement}

\author{
Cáris de Rezende Pena ${ }^{(1)}$, Gustavo Lamego de Barros Costa $^{(1)}$, Ana Paula Monteiro Gomides ${ }^{(2)}$, \\ Fabiana Britto Goulart ${ }^{(3)}$, Daniele Linhares Coelho ${ }^{(4)}$, Maria Vitória Quintero ${ }^{(3)}$
}

\section{RESUMO}

Sarcoidose é uma doença granulomatosa sistêmica de ocorrência rara em crianças. Vasculite associada à sarcoidose é incomum e pode acometer vasos de pequeno a grande calibres. Envolvimento sintomático do sistema nervoso central e periférico ocorre em $5 \%$ a $10 \%$ dos pacientes e acometimento ósseo em 3\% a 13\%. Sarcoidose do trato geniturinário é extremamente rara. Descrevemos o caso de uma criança de 12 anos com diagnóstico de sarcoidose associada à vasculite, acometimento do sistema nervoso central, ósseo e genital. O amplo espectro de manifestações clínicas raras neste caso ilustra o caráter multissistêmico e multifacetário da sarcoidose.

Palavras-chave: sarcoidose, sarcoidose na infância, tumores testiculares, doenças granulomatosas.

\section{INTRODUÇÃO}

Sarcoidose é uma doença granulomatosa sistêmica de ocorrência rara na infância. Em crianças menores de cinco anos, apresenta-se comumente com artrite, rash cutâneo, uveíte e linfadenopatia sem acometimento pulmonar. Acima desta idade o quadro se assemelha a doença em adultos, com envolvimento pulmonar presente em até 90\% dos pacientes. Vasculite associada à sarcoidose é incomum, podendo acometer vasos de pequenos a grandes calibres. Envolvimento sintomático do sistema nervoso central e

\begin{abstract}
Sarcoidosis is a systemic granulomatous disease with rare occurrence in children. Vasculitis associated to sarcoidosis is uncommon and can affect small, medium or large-sized vessels. Symptomatic involvement of the central nervous and peripheral system occurs in $5 \%$ to $10 \%$ of the patients and osseous in 3\% to $13 \%$. Sarcoidosis of the genitourinary tract is extremely rare. We describe a case of a 12-year-old child with a diagnosis of sarcoidosis associated with vasculitis and with involvement of the central nervous system, osseous and genital. This case illustrates the wide spectrum of rare clinical manifestations in sarcoidosis.
\end{abstract}

Keywords: sarcoidosis, sarcoidosis in the childhood, testicular tumor, granulomatous disease.

periférico ocorre em $5 \%$ a $10 \%$ dos pacientes e acometimento ósseo em 3\% a 13\%. Sarcoidose do trato geniturinário é extremamente rara, com poucos casos descritos na literatura referindo envolvimento testicular.

\section{RELATO DE CASO}

Criança de 12 anos, sexo masculino, negra, iniciou aos dez anos de idade artralgia acometendo punhos, cotovelos, joelhos e tornozelos, edema em membros inferiores e emagrecimento. Evoluiu com dor e aumento testicular

\footnotetext{
* Trabalho realizado no Serviço de Reumatologia da Santa Casa de Belo Horizonte, Centro de Pós-Graduação e Pesquisa da Faculdade de Ciências Médicas de Minas Gerais (FCMMG), Belo Horizonte, MG, Brasil. Recebido em 14/10/2004. Aprovado, após revisão, em 04/05/2005.

1. Reumatologista pela Santa Casa de Belo Horizonte, Centro de Pós-Graduação e Pesquisa da FCMMG.

2. Reumatologista do Serviço de Reumatologia da Santa Casa de Belo Horizonte, Centro de Pós-Graduação e Pesquisa da FCMMG.

3. Reumatologista pediátrica do Serviço de Reumatologia da Santa Casa de Belo Horizonte, Centro de Pós-Graduação e Pesquisa da FCMMG.

4. Especializanda em Reumatologia Pediátrica pelo Serviço de Reumatologia da Santa Casa de Belo Horizonte, Centro de Pós-Graduação e Pesquisa da FCMMG.

Endereço para correspondência: Dra. Cáris de Rezende Pena. Santa Casa de Belo Horizonte, Centro de Pós-Graduação e Pesquisa da Faculdade de Ciências Médicas de Minas Gerais (FCMMG). Rua Monte Sião, 334/302, Serra, CEP 30240-050. Belo Horizonte, MG, Brasil. E-mail: carisrpena@ig.com.br 
esquerdo e paresia no membro inferior esquerdo. Ao exame apresentava-se abaixo do percentil para idade em peso e estatura, hipocorado, pele ressecada com áreas de descamação, pápulas hiperpigmentadas em região cervical anterior e posterior, linfadenomegalia cervical e inguinal indolor e móvel, lesões nodulares dolorosas em membros inferiores, edema em dorso da mão esquerda e em terço distal dos membros inferiores, doloroso e não depressível, com dor à mobilização dos tornozelos, punhos e joelhos. Observado aumento testicular esquerdo doloroso com espessamento do funículo espermático e hemiparesia distal grau IV à esquerda. Avaliação oftalmológica evidenciou sinéquias posteriores em ambos os olhos.

Propedêutica detectou anemia microcítica hipocrômica (Hb: 8,6), leucograma, plaquetas, função renal, hepática, tireoidiana e urina rotina normais, Fe sérico e saturação de transferrina baixos, VHS $18 \mathrm{~mm} / 60 \mathrm{~min}$ (referência até 20 $\mathrm{mm} / 60$ "), PCR 48 (referência < 6), hipoalbuminemia e hipergamaglobulinemia, FAN, FR, ANCA P e C negativos, hemocultura negativa, cálcio total, fósforo e fosfatase alcalina normais, presença de $\mathrm{HbC}(38,8 \%)$ à eletroforese de hemoglobina, pesquisa de BAAR em cotovelos e lóbulo de orelha negativa, PPD não reator em junho de 2002 (último reforço da BCG feito em maio de 2002), sorologias para rubéola, toxoplasmose, blastomicose e HIV negativas.

Radiografia das mãos mostrava aumento de partes moles em metacarpo da mão direita e reação periosteal em segundo metacarpo direito (Figura 1). Radiografia de ossos longos dos membros inferiores, bacia, coluna torácica e lombar com áreas osteopênicas circunscritas, áreas focais múltiplas de esclerose óssea, trabeculado ósseo esparso e grosseiro (Figura 2). Ultra-som e tomografia computadorizada (TC) de abdome evidenciaram linfadenopatia mesentérica e peri-cava. TC tórax normal e TC de crânio com área isquêmica em substância branca à direita. Biópsia de linfonodo cervical mostrou linfadenite crônica reacional inespecífica e biópsia de nódulo nos membros inferiores perivasculite dérmica, hipodérmica e paniculite, configurando eritema nodoso. Achado de mielite granulomatosa de etiologia não determinada à biópsia de crista ilíaca com pesquisa de BAAR e fungos negativa (Figura 3).

O paciente foi submetido à exploração cirúrgica testicular sendo encontrado tumoração em epidídimo invadindo canal inguinal. Realizado orquiectomia em razão de suspeita de lesão neoplásica. Estudo anatomopatológico evidenciou numerosos granulomas constituídos por histiócitos epitelióides, algumas células gigantes e linfócitos, sem necrose central e fibrinóide, com pesquisa para BAAR e fungos

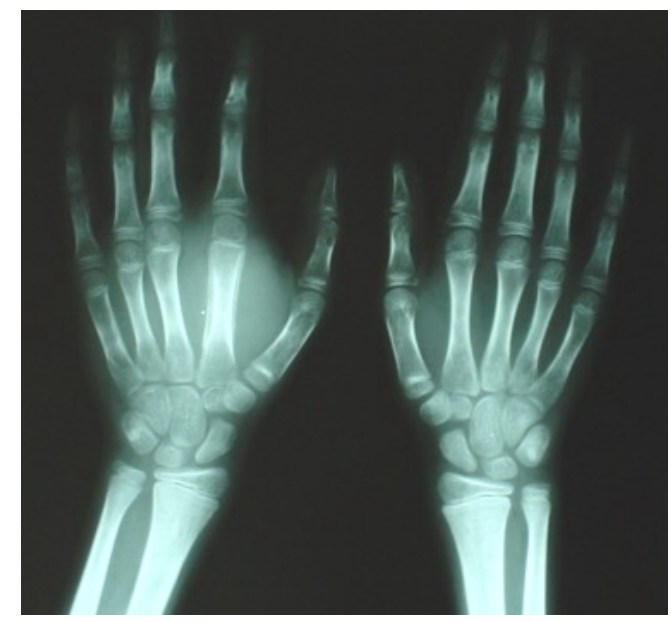

FIgURA 1 - Radiografia de mãos mostrando reação periosteal em segundo metacarpo da mão direita

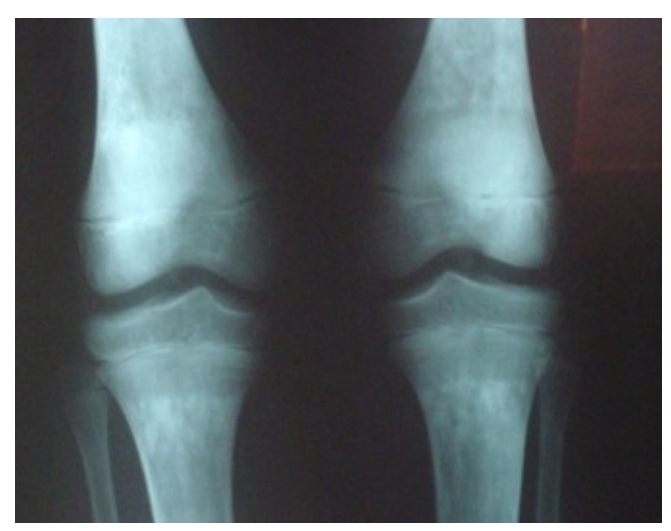

FiguRa 2 - Radiografia de ossos longos de membros inferiores mostrando áreas osteopênicas circunscritas, áreas focais múltiplas de esclerose óssea, trabeculado ósseo esparso e grosseiro

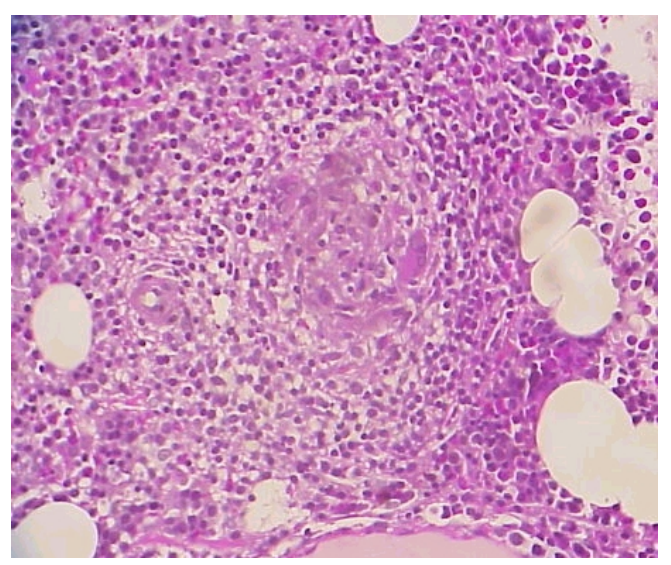

Figura 3 -Corte de medula óssea evidenciando granuloma 
negativa. Vasos (artérias e veias) continham infiltrado histiocitário intimal e perivascular com células gigantes e rompimento das limitantes elásticas interna e externa (Figuras 4 e 5). Diante dos dados clínicos e do estudo anatomopatológico fez-se o diagnóstico de sarcoidose associada à vasculite. Foi iniciado prednisona $1 \mathrm{mg} / \mathrm{kg} /$ dia e metotrexato $0,5 \mathrm{mg} / \mathrm{kg} / \mathrm{semana}$, que foram usados irregularmente pelo paciente. O mesmo evoluiu com acometimento testicular direito que remitiu após adequação do uso das medicações.

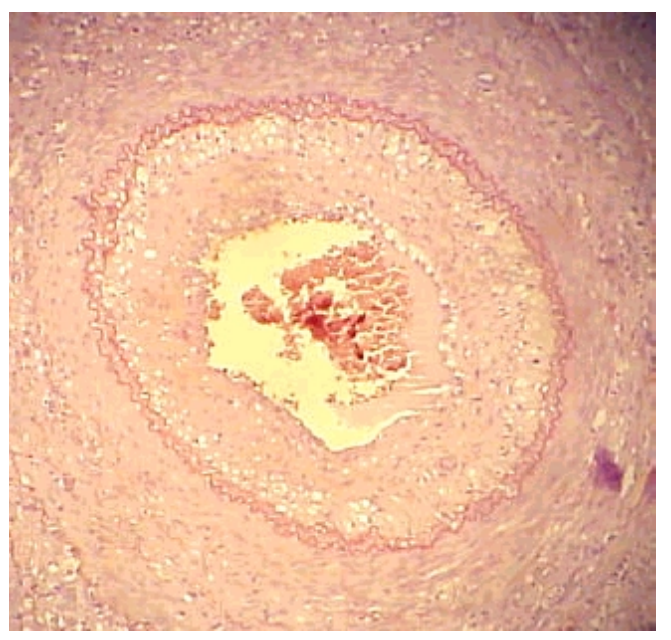

Figura 4 - Corte de testículo. Vaso com duplicação de lâmina elástica evidenciando vasculite

\section{DISCUSSÃO}

O diagnóstico de sarcoidose associada a vasculite neste paciente foi firmado pelo achado de granulomas não caseosos com células epitelióides, infiltrado inflamatório perivascular e intimal com rompimento da lâmina elástica dos vasos ao estudo anatomopatológico da massa testicular, associado aos achados clínico-laboratoriais. Clinicamente, o paciente apresentava emagrecimento, anergia cutânea (PPD não reator), lesões cutâneas secundárias à perivasculite, linfadenopatia, poliartralgia, edema de partes moles, seqüela de uveíte anterior bilateral e envolvimento testicular. Não apresentava evidências clínicas e radiológicas de acometimento pulmonar. Alterações em ossos longos, coluna lombar e bacia foram detectadas às radiografias.

Vasculite associada à sarcoidose é uma complicação rara. Vasos de pequeno a grande calibre podem ser envolvidos mimetizando vasculite leucocitoclástica, poliarterite nodosa, poliangiíte microscópica e arterite de Takayasu ${ }^{(1-5)}$. Duas formas de acometimento vascular são reconhecidas. $\mathrm{Na}$ primeira, os granulomas causam destruição dos vasos sanguíneos por compressão extrínseca, sendo acometidas tanto artérias quanto veias. Rompimento da lâmina elástica nesta forma pode ser causado por infiltrado inflamatório crônico associado ao granuloma, na ausência de verdadeira necrose vascular. A segunda forma é denominada angiíte sarcóide ou granulomatose sarcóide necrotizante e é considerada uma variante da sarcoidose. Nesta, a vasculite é causada diretamente pelos granulomas e o rompimento das lâminas elásticas ocorre por verdadeira necrose vascular. O acometimento pulmonar é muito comum, enquanto o acome-
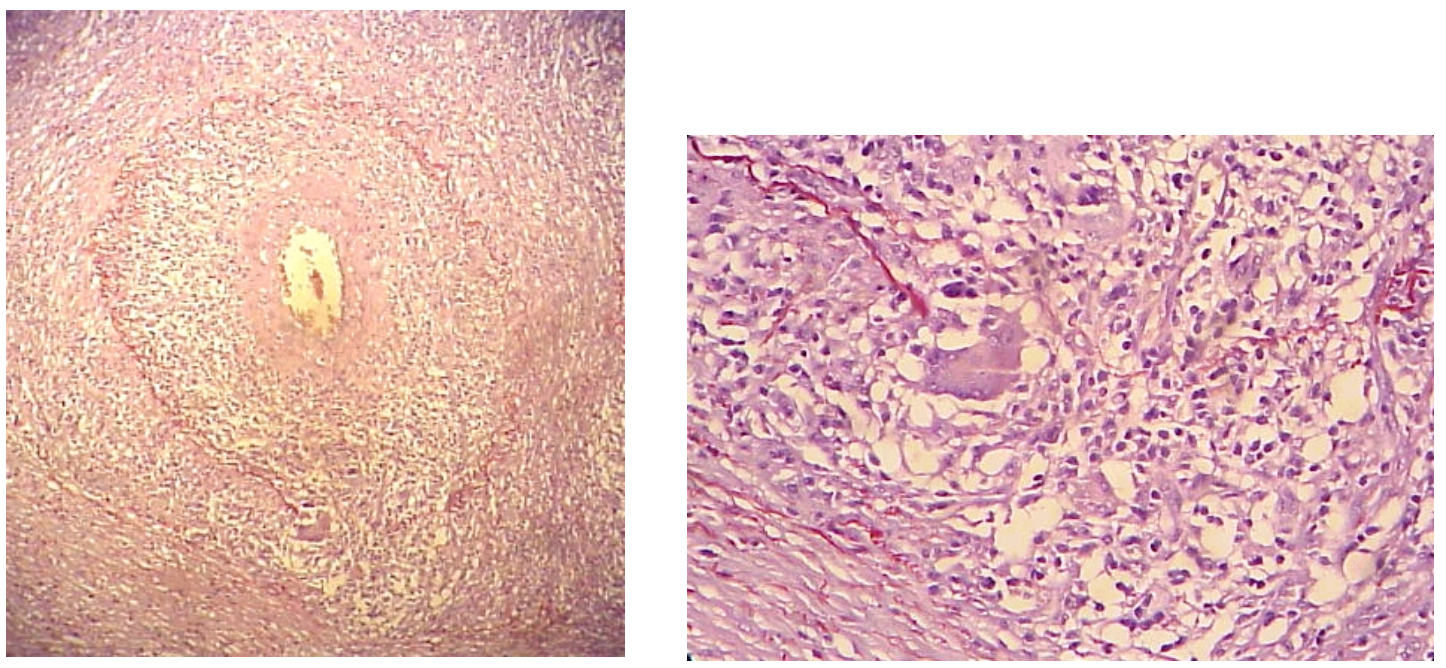

Figura 5 - Corte de testículo evidenciando rompimento da lâmina elástica por granuloma e infiltrado inflamatório. Ao lado, detalhe da figura 
timento linfonodal e extrapulmonar são menos freqüentes. O paciente apresentava achados clínicos (acometimento extrapulmonar) e ao estudo anatomopatológico (vasculite sem necrose, com acometimento de artérias e veias) compatíveis com a primeira forma descrita.

Envolvimento sintomático do sistema nervoso central e periférico ocorre em aproximadamente 5\% a 10\% dos pacientes e é associado a mau prognóstico ${ }^{(6,7)}$. Pode ocorrer acometimento isolado de pares cranianos, mais frequentemente do sétimo par, da leptomeninge, hipotálamo, hipófise, massas cranianas e neuropatia periférica. O paciente apresentou quadro isquêmico tendo como seqüela hemiparesia esquerda, provavelmente por neurosarcoidose.

Acometimento ósseo na sarcoidose $\mathrm{e}^{(5,7,8)}$ ocorre em 3\% a 13\% dos pacientes, mais comumente em indivíduos de ascendência africana, implica em doença crônica e grave sendo fator de mau prognóstico. O envolvimento geralmente é assintomático. Pode haver edema de tecidos moles e aumento da sensibilidade local acompanhando as lesões ósseas subjacentes. Estas ocorrem principalmente em mãos e pés, nas porções média e distal das falanges e metacarpos, com uma distribuição bilateral, assimétrica e predominantemente no osso cortical, preservando o periósteo. Envolvimento de ossos do crânio, costelas, bacia, vértebras e periósteo é incomum. À radiografia as lesões aparecem como áreas líticas sob a forma de pequenos defeitos corticais com aparência rendilhada (ou reticular) ou lesões maiores em "saca bocado", lesões permeativas caracterizadas por tuneli-

\section{REFERÊNCIAS}

1. Fernandes SRM, Singsen BH, Hoffman GS: Sarcoidosis and systemic vasculitis. Semin Arthritis and Reum 30: 33-46, 2000.

2. Kwong T, Valderrama E, Paley C, Ilowite N: Systemic necrotizing vasculitis associated with childhood sarcoidosis. Semin Arthritis Rheum 23: 388-95, 1994.

3. Petri M, Barr E, Cho K, Farmer E: Overlap of granulomatous vasculitis and sarcoidosis: presentation with uveitis, eosinophilia, leg ulcers, sinusitis and past foot drop. Jornal of Rheumatol 15: 1171-3, 1998.

4. Watson I, Hill CM, Biggart JD, Russel CJ, Garrett PJ: Sarcoidosis and primary systemic vasculitis. Nephrol Dial Transplant 11: 1631-3, 1996.

5. Abril A, Cohen MD: Rheumatologic manifestations of sarcoidosis. Curr Opin Rheumatol 16: 51-5, 2003.

6. [No authors listed]. Statement on sarcoidosis. Joint Statement of the American Thoracic Society (ATS), the European Respiratory Society (ERS) and the World Association of Sarcoidosis and Other Granulomatous Disorders (WASOG) adopted by the ATS Board of Directors and by the ERS Executive Committee, February 1999. Am J Respir Crit Care Med 160: 736-55.1999. zação subcortical (luscências longitudinais) e lesões destrutivas com fraturas e seqüestro ósseo. Menos comumente há osteoesclerose generalizada da coluna, pelve, costelas e ossos do crânio. O paciente em questão apresentava edema de partes moles em mãos e membros inferiores, periostite, osteoesclerose e acometimento de sítios incomuns como bacia e vértebras.

O sistema reprodutor masculino é raramente afetado na sarcoidose $\mathrm{e}^{(6,7)}$. Encontramos na literatura poucos relatos de caso $^{(9-14)}$ nos quais, em pacientes submetidos à propedêutica de massa paratesticular, foi diagnosticado sarcoidose. Kodama K et al. ${ }^{(13)}$ relatam somente 59 casos descritos na literatura de sarcoidose com acometimento do trato genital comprovado histologicamente. Nosso paciente cursou com envolvimento epididimal bilateral.

Concluindo, apresentamos o caso clínico de um paciente com amplo espectro de manifestações raras e de mau prognóstico, ilustrando o caráter multissistêmico da sarcoidose. Entre as manifestações raras encontram-se vasculite, neurosarcoidose, acometimento ósseo e do trato geniturinário. Entre os fatores de mau prognóstico incluem-se raça negra, uveíte crônica, lesões ósseas císticas e neurosarcoidose.

\section{AGRADECIMENTOS}

Ao Dr. Roberto Junqueira de Alvarenga, pela contribuição no diagnóstico anatomopatológico.

7. West SG, Kotzin BL: Sarcoidosis. Rheumatology 2: 1735-45, 2003.

8. Wilcox A, Bharadwaj P, Sharma OP: Bone Sarcoidosis. Curr Opin Rheumatol 12: 321-30, 2000.

9. Berkowitz JM, Unger P, Fyfe B: Sarcoidosis involving a paratesticular adenomatoid tumor. Sarcoidosis Vasc Diffuse Lung Dis 13: 183-5, 1996.

10. Esser R, Rothenber KH: Sarcoidosis of the spermatic cord and epididymus. Aktuelle Urol 34: 354-5, 2003.

11. Evans SS, Fisher RG, Scott MA, Kennedy BG, Brock JW, Wilson GJ: Sarcoidosis presenting as bilateral testicular masses. Pediatrics 100: 392-4, 1997.

12. Metcalfe MS, Rees Y, Morgan P, O'reilly K, Shandu DPS: Sarcoidosis presenting as a testicular mass. BJU Internat 82: 769, 1998.

13. Kodama K, Hasegawa T, Egawa M, Tomosugi N, Mukai A, Namiki M: Bilateral epididymal sarcoidosis presenting without radiografic evidence of intrathoracic lesion: Review of sarcoidosis involving the male reproductive tract. Internat J Urol 11: 345, 2004.

14. Hurd DS, Olsen T: Cutaneous sarcoidosis presenting as a testicular mass. Cutis 66: 435-8, 2000. 